\title{
SOME CURVATURE PROPERTIES OF LOCALLY CONFORMAL KÄHLER MANIFOLDS
}

BY

\author{
IZU VAISMAN
}

\begin{abstract}
Curvature identities and holomorphic sectional curvature of locally conformal Kähler manifolds are investigated. Particularly, sufficient conditions for such manifolds to be globally conformal Kähler are derived.
\end{abstract}

In [2], A. Gray showed the importance of various curvature identities in the geometry of the almost Hermitian manifolds. It is therefore to be expected that such identities should play some role in the geometry of the locally conformal Kähler (1.c.K.) manifolds [4], [5], too. Particularly, we can ask whether such identities could characterize the Kähler manifolds in the class of the l.c.K. manifolds and we want to give here a theorem of this kind. We shall also obtain information about the Kähler-nullity distribution [2] and about the holomorphic sectional curvature of the l.c.K. manifolds.

1. L.c.K. manifolds. A. Gray studied in [2] various curvature identities which could appear in the almost-Hermitian geometry. As usual, we are working in the $C^{\infty}$-category, and we shall denote by $M^{2 n}(n>1)$ the manifold, by $J$ its complex structure, by $g$ the Hermitian metric and by $\Omega(X, Y)=g(X, J Y)(X, Y, \ldots$ are always vector fields on $M$ ) its fundamental form. We refer, for instance, to [3] for a detailed exposition of the almost Hermitian geometry.

Now, if $\mathcal{L}$ is some class of almost-Hermitian manifolds, Gray defines the classes $\mathscr{C}_{i}(i=1,2,3)$ as its subclasses characterized respectively by the identities [2]:

(1) $R_{X Y Z W}=R_{X Y(J Z)(J W)}$,

(2) $R_{X Y Z W}-R_{(J X)(J Y) Z W}-R_{(J X) Y(J Z) W}-R_{(J X) Y Z(J W)}=0$,

(3) $R_{X Y Z W}=R_{(J X)(J Y)(J Z)(J W)}$,

where $R$ is the curvature tensor of the Levi-Civita connection $\nabla$ of $g$. It is known that for every $\mathcal{L}$ one has $\mathcal{L}_{1} \subseteq \mathscr{L}_{2} \subseteq \mathcal{L}_{3} \subseteq \mathcal{L}$ and that the class $\mathcal{K}$ of the Kähler manifolds satisfies all of the relations (1), (2), (3) [2].

Particularly, if we denote by $\mathcal{E}$ the class of the l.c.K. manifolds, we can speak of the classes $\mathcal{E} \mathcal{K}_{i}(i=1,2,3)$ and we shall investigate some relations between these classes.

But, before doing this, we recall some properties of the manifolds of $\mathcal{E} \mathcal{K}$ and we establish some useful results including the determination of their Kähler-nullity distribution [2]. More about this subject can be found in [5].

Received by the editors January 8, 1979 and, in revised form, June 10, 1979.

1980 Mathematics Subject Classification. Primary 53C55. 
First, an almost-Hermitian manifold $M$ is 1.c.K. iff

$$
[J, J]=0, \quad d \Omega=\omega \wedge \Omega,
$$

where $[J, J]$ is the Nijenbuis tensor of $J[3]$ and

$$
\omega=[1 /(n-1)] \delta \Omega \circ J=[1 /(n-1)] \Lambda d \Omega
$$

is the so-called Lee form and is closed ( $\delta$ is the codifferential and $\Lambda=i(\Omega)$ ).

Since $\omega$ is closed, we have locally $\omega=d \sigma$ and

$$
g^{\prime}=e^{-\sigma} g
$$

are the local conformal Kähler metrics of $g$. One can see that the Levi-Civita connection of the metrics $g^{\prime}$ define a global connection $\tilde{\nabla}$ on $M$, which is given by

$$
\tilde{\nabla}_{X} Y=\nabla_{X} Y-\frac{1}{2} \omega(X) Y-\frac{1}{2} \omega(Y) X+\frac{1}{2} g(X, Y) B,
$$

where $B$ is the contravariant field of $\omega$ called the Lee field. We call $\tilde{\nabla}$ the Weyl connection and it is shown in [6] that the conditions (1.1) are equivalent with

$$
\tilde{\nabla}_{X} J=0 \text {. }
$$

Of course, $\nabla$ is no more an almost complex connection and we have

$$
\left(\nabla_{X} J\right)(Y)=-\frac{1}{2} g(X, Y) A-\frac{1}{2} \Omega(X, Y) B+\frac{1}{2} \theta(Y) X-\frac{1}{2} \omega(Y) J X,
$$

where

$$
\theta=\omega \circ J, \quad A=-J B .
$$

As a consequence, we see that

$$
\Phi(X, Y)=-2 \omega\left(\left(\nabla_{X} J\right)(Y)\right)
$$

is an exterior 2-form on $M$ and satisfies

$$
\Phi=|\omega|^{2} \Omega-\omega \wedge \boldsymbol{\theta} .
$$

To get another interesting expression of this form, we consider

$$
L_{B} \Omega=i(B) d \Omega+d i(B) \Omega=i(B)(\omega \wedge \Omega)+d \theta,
$$

which gives

$$
L_{B} \Omega=|\omega|^{2} \Omega-\omega \wedge \theta+d \theta
$$

and therefore

$$
\Phi=L_{B} \Omega-d \theta .
$$

In [2], the Kähler-nullity distribution is defined as the function $m \mapsto \mathcal{K}(m)$ sending every $m \in M$ to

$$
\mathscr{K}(m)=\left\{X \in T_{m} M /\left(\nabla_{X} J\right)(Y)=0 \text { for all } Y\right\} .
$$

Clearly, this may have singular points.

In our case, we prove

Proposition 1.1. If $M \in \mathcal{C} \mathcal{K}$, the Kähler-nullity distribution $\mathcal{K}(M)$ is equal to the annihilator of the 2-form $\Phi$. Its singular points are the vanishing points of $\omega$ and at such a point $\mathscr{K}(m)=T_{m} M$. At every nonsingular point $m, \mathcal{K}(m)$ is two-dimensional and is generated by $A$ and $B$. 
Proof. It is clear from the definitions of $\mathcal{K}(M)$ and $\Phi$ that $\mathcal{K}(M)$ is included in the annihilator, Ann $\Phi$, defined by the $X$ satisfying $\Phi(X, Y)=0$ for every $Y$.

Now, if at $m \in M, \omega(m)=0$, we have $\operatorname{Ann}_{m} \Phi=T_{m} M$ and, at the same time, from (1.6), $\mathcal{K}(m)=T_{m} M$. I.e., $\mathcal{K}(m)=\mathrm{Ann}_{m} \Phi$ as stated.

If, on the contrary, $\omega(m) \neq 0$, we note first that $A$ and $B$ belong to both $\mathscr{K}(m)$ and $\mathrm{Ann}_{m} \Phi$, which is an easy consequence of (1.6) and (1.8). On the other hand, we can see that $\operatorname{rank}_{m} \Phi=2(n-1)$. In fact, since $A$ and $B$ are linearly independent and belong to Ann $\Phi$, we have $\operatorname{rank} \Phi \leqslant 2(n-1)$. But if we had $\operatorname{rank} \Phi<$ $2(n-1),(1.9)$ would imply rank $\Omega<2 n$, which is false. This shows that $A_{n n} \Phi$ $\subseteq \mathcal{K}(m)$ and we are done.

It is also worthwhile to consider the complementary orthogonal distribution $\mathscr{K}^{\perp}(M)$ of $\mathscr{K}(M)$ and we call it the Kähler distribution of $M$. If $\omega \neq 0$ everywhere, $K^{\perp}(M)$ has the equations

$$
\omega=0, \quad \theta=0 .
$$

2. Curvature identities. We go over now to curvature considerations. These will be based on the relation between the curvature tensor of the connections $\nabla$ and $\tilde{\nabla}$, which is familiar in conformal Riemannian geometry. Namely, from

$$
R_{X Y}=\nabla_{X} \nabla_{Y}-\nabla_{Y} \nabla_{X}-\nabla_{[X, Y]}
$$

and from (1.4) we get

$$
\begin{aligned}
\tilde{R}_{X Y} Z=R_{X Y} Z-\frac{1}{2}\{[ & \left.\left(\nabla_{X} \omega\right)(Z)+\frac{1}{2} \omega(X) \omega(Z)\right] Y \\
& -\left[\left(\nabla_{Y} \omega\right)(Z)+\frac{1}{2} \omega(Y) \omega(Z)\right] X \\
& \left.-g(Y, Z)\left[\nabla_{X} B+\frac{1}{2} \omega(X) B\right]+g(X, Z)\left[\nabla_{Y} B+\frac{1}{2} \omega(Y) B\right]\right\} \\
& -\left(|\omega|^{2} / 4\right)\{g(Y, Z) X-g(X, Z) Y\} .
\end{aligned}
$$

Put

$$
L(X, Y)=\left(\nabla_{X} \omega\right)(Y)+\frac{1}{2} \omega(X) \omega(Y)=g\left(\nabla_{X} B, Y\right)+\frac{1}{2} \omega(X) \omega(Y),
$$

where the last equality follows from $\left(\nabla_{X} g\right)(B, Y)=0$.

Since $\omega$ is closed, $L$ is a symmetric 2-tensor and, by scalar multiplication of (2.1) by $W$, we get the well-known formula [1]

$$
\begin{aligned}
& e^{o} R_{X Y Z W}^{\prime}=R_{X Y Z W}-\frac{1}{2}\{ L(X, Z) g(Y, W)-L(Y, Z) g(X, W) \\
&+L(Y, W) g(X, Z)-L(X, W) g(Y, Z)\} \\
&-\left(|\omega|^{2} / 4\right)\{g(Y, Z) g(X, W)-g(X, Z) g(Y, W)\},
\end{aligned}
$$

where $R^{\prime}$ is the curvature tensor of the local Kähler metric $g^{\prime}=e^{-\sigma} g$.

THEOREM 2.1. (i) If $M \in \mathcal{C} \mathscr{K}_{1}$ is compact, then $M$ is a Kähler manifold. If compactness is replaced by the condition $\operatorname{div} B \leqslant 0$, the same conclusion prevails.

(ii) $\mathcal{C} \mathcal{K}_{2}=\mathcal{C} \mathcal{K}_{3}[2]$, and every manifold of this class for which either $B$ is analytic or the trajectories of $A$ and $B$ are geodesic lines is a Kähler manifold. 
Proof. (i) We replace in (1) of $\S 1$ the tensor $R$ by the help of (2.3) and use the fact that $R^{\prime}$ is the curvature of a Kähler metric, hence it satisfies the same identity (1). If in the obtained relation we go over to local components we get

$$
\begin{aligned}
g_{j e} L_{i k}-g_{i e} L_{j k}+ & g_{i k} L_{j e}-g_{j k} L_{i e}+\left(|\omega|^{2} / 2\right)\left\{g_{j k} g_{i e}-g_{i k} g_{j e}\right\} \\
= & \Omega_{j e} J_{k}^{s} L_{s i}-\Omega_{i e} J_{k}^{s} L_{s j}+\Omega_{i k} J_{e}^{s} L_{s j}-\Omega_{j k} J_{e}^{s} L_{s i} \\
& +\left(|\omega|^{2} / 2\right)\left\{\Omega_{j k} \Omega_{i e}-\Omega_{i k} \Omega_{j e}\right\} .
\end{aligned}
$$

Next, we contract (2.4) with $g^{j e} g^{i k}$ and use the relation between $g, J, \Omega$ as well as the Hermitian character of $g$. We shall obtain thereby

$$
2 g^{i j} L_{i j}=n|\omega|^{2} \text {. }
$$

Finally, replacing $L_{i j}$ from (2.2) yields

$$
2 \operatorname{div} B-(n-1)|\omega|^{2}=0,
$$

which proves directly that $M$ is Kähler $(\omega=0)$ if $\operatorname{div} B \leqslant 0$, and proves the same result by integrating over $M$, if $M$ is compact, since $\int_{M} \operatorname{div} B=0$.

(ii) In a similar manner, we find the following characteristic relation of $e \mathcal{K}_{3}$ :

$$
g_{i k} P_{j e}-g_{j k} P_{i e}-g_{i e} P_{j k}+g_{j e} P_{i k}=0 \text {, }
$$

where the tensor $P$ is defined by

$$
P(X, Y)=L(X, Y)-L(J X, J Y) .
$$

Now by contracting in (2.7) with $g^{i k}$ and next, in the resulting relation, with $g^{j e}$, we see that (2.7) is equivalent with $P=0$, i.e. $\odot \mathcal{K}_{3}$ is characterized by

$$
L(X, Y)=L(J X, J Y)
$$

( $L$ is a Hermitian form).

Furthermore, we get similarly for the class $\mathrm{CK}_{2}$ the characteristic relation

$$
g_{j e} P_{i k}-g_{j k} P_{i e}+g_{e m} J_{i}^{m} J_{j}^{s} P_{s k}-g_{k m} J_{i}^{m} J_{j}^{s} P_{s e}=0 \text {, }
$$

which obviously yields $\mathrm{e} \mathcal{K}_{3} \subseteq \mathrm{e} \mathcal{K}_{2}$ and, since also $\mathrm{C} \mathcal{K}_{2} \subseteq \mathrm{e} \mathcal{K}_{3}$, we are done for the first assertion of (ii). (See also Theorem 5.1 of [2].)

Finally, let us take $M \in \mathcal{C} \mathcal{K}_{3}$, i.e. satisfying (2.9) or equivalently (see (2.2)),

$$
g\left(\nabla_{X} B, Y\right)-g\left(\nabla_{J X} B, J Y\right)+\frac{1}{2} \omega(X) \omega(Y)-\frac{1}{2} \theta(X) \theta(Y)=0 .
$$

Here we shall use

$$
\begin{aligned}
\nabla_{J X} B & =\nabla_{B}(J X)-[B, J X]=\left(\nabla_{B} J\right)(X)+J\left(\nabla_{B} X\right)-[B, J X] \\
& =J\left(\nabla_{B} X\right)-[B, J X],
\end{aligned}
$$

where the last equality is valid since, by Proposition $1.1, B$ belongs to the Kähler-nullity distribution $\mathscr{K}(M)$.

As a consequence, (2.11) becomes

$$
g([B, J X]-J[B, X], J Y)+\frac{1}{2}(\omega(X) \omega(Y)-\theta(X) \theta(Y))=0 .
$$

If $B$ is an analytic vector field, (2.12) gives $\omega(X) \omega(Y)=\theta(X) \theta(Y)$, which is easily seen to hold iff $\omega=0$, i.e. in the case when $M$ is Kähler: 
As for the last statement of the theorem, note that for $X=B,(2.12)$ reduces to

$$
g([B, A], J Y)=\left(|\omega|^{2} / 2\right) \omega(Y)=\left(|\omega|^{2} / 2\right) g(B, Y)
$$

whence $2 J[B, A]=-|\omega|^{2} B$ or

$$
[A, B]=\left(|\omega|^{2} / 2\right) A
$$

In other words, the Kähler-nullity distribution of $M \in \mathcal{C} \mathcal{K}_{3}$ is integrable.

But (2.14) means

$$
\left(|\omega|^{2} / 2\right) A=\nabla_{A} B-\nabla_{B} A=\nabla_{A}(J A)+\nabla_{B}(J B)=J\left(\nabla_{A} A+\nabla_{B} B\right),
$$

since both $A$ and $B$ belong to the Kähler-nullity distribution of $M$.

Hence, if the trajectories of $A$ and $B$ are geodesic lines we have $A=0$, i.e. $\omega=0$. Q.E.D.

REMARKS. (1) Of course, for $M$ to carry a Kähler metric, it suffices that the hypotheses of Theorem 2.1 be satisfied for a metric which is globally conformal to the given one.

(2) The basic example of the Hopf manifolds $H \in \mathcal{C} \mathscr{K}$ [5], [6] shows that the class $\mathrm{CK}_{3}$ is strictly included in $\mathcal{C} \mathscr{K}$. Indeed, in this case, $B$ is analytic but the manifold is not Kähler whence, by the above Theorem 2.1, we have $H \bar{E} \mathcal{E} \mathcal{K}_{3}$ and, of course, $H \bar{E} \odot \mathscr{K}_{i}(i=1,2,3)$.

(3) It seems to us that there is no chance to find a curvature identity for the class $\mathcal{C K}_{\mathcal{K}}$ which would be simpler than the general Hermitian identity of [2]. However this is still an open problem.

3. Holomorphic sectional curvature. In this section, we shall derive some information about the holomorphic sectional curvature (h.s.c.) of an l.c.K. manifold and we begin by

Proposition 3.1. Let $M$ be a complete l.c.K. manifold and assume that its h.s.c. satisfies the condition

$$
K_{X(J X)} \geqslant\left(|\omega|^{2} / 4\right)+\delta
$$

for some $\delta>0$. Then $M$ is compact simply connected and, hence, globally conformal Kähler.

Proof. This is a straightforward consequence of Theorems 8.1, 8.2 of [2], which say that a complete almost-Hermitian manifold for which

$$
K_{X(J X)}-\left|\left(\nabla_{X} J\right)(X)\right|^{2}|X|^{-4} \geqslant \delta>0,
$$

is compact and simply connected. (See [2], [3] for the definition and the notation of the holomorphic sectional curvature $K_{X(J X)}$.)

In our case, we have from (1.6)

$$
\left|\left(\nabla_{X} J\right)(X)\right|^{2}|X|^{-4}=\frac{1}{4}\left\{|\omega|^{2}-\left([\omega(X)]^{2}+[\theta(X)]^{2}\right)|X|^{-2}\right\}
$$

which leads to the fact that $M$ is compact simply connected. Hence $\omega$ is an exact form and this implies also that $M$ is globally conformal Kähler [2]. 
Further information will be contained in

THEOREM 3.2. Let $M$ be an l.c.K. manifold with metric $g$ and local Kähler metrics $g^{\prime}$. Then

(i) if the metrics $g^{\prime}$ have nonpositive h.s.c. and if for every $X, d \theta(X, J X)<0, g$ has also nonpositive h.s.c.,

(ii) if the metrics $g^{\prime}$ have nonnegative h.s.c. and if for every $X,\left(L_{B} \Omega\right)(X, J X)>0$, $g$ has also nonnegative h.s.c.,

(iii) if the hypotheses of (ii) are strengthened by asking that for all $X$,

$$
\left(L_{B} \Omega\right)(X, J X)|X|^{-2} \geqslant \delta>0,
$$

then $M$ is a compact simply connected globally conformal Kähler manifold.

Proof. A straightforward application of formula (2.3) yields

$$
e^{-\sigma} K_{X(J X)}^{\prime}=K_{X(J X)}-\frac{|\omega|^{2}}{4}+\frac{L(X, X)+L(J X, J X)}{2|X|^{2}},
$$

where $K^{\prime}$ is the sectional curvature of $g^{\prime}$.

Consider

$$
\lambda(X, Y)=L(X, J Y)-L(J X, Y),
$$

which is a 2 -form because of the symmetry of $L$. This is interesting since we have

$$
L(X, X)+L(J X, J X)=-\lambda(X, J X) \text {. }
$$

From (3.4), it follows

$$
\lambda(X, Y)=\left(\nabla_{X} \omega\right)(J X)-\left(\nabla_{J X} \omega\right)(Y)+\frac{1}{2}(\omega \wedge \theta)(X, Y)
$$

where we shall: (a) explicitate the first term, (b) replace the second term by $-\left(\nabla_{Y} \omega\right)(J X)$ and explicitate, and (c) replace

$$
\nabla_{X}(J Y)=\left(\nabla_{X} J\right)(Y)+J\left(\nabla_{X} Y\right), \quad \nabla_{Y}(J X)=\left(\nabla_{Y} J\right)(X)+J\left(\nabla_{Y} X\right) .
$$

These operations lead to

$$
\lambda=d \theta+\frac{1}{2} \omega \wedge \theta+\Phi,
$$

where $\Phi$ is defined by (1.8). Or, using formula (1.9), we obtain

$$
\lambda=|\omega|^{2} \Omega-\frac{1}{2} \omega \wedge \theta+d \theta \text {. }
$$

Now, if we use (3.2), (3.3), (3.5) and (3.7), we get

$$
e^{-\sigma} K_{X(J X)}^{\prime}=K_{X(J X)}+\left|\left(\nabla_{X} J\right)(X)\right|^{2}|X|^{-4}-d \theta(X, J X) / 2|X|^{2}
$$

and, clearly, this formula proves (i).

Next, replace $\Phi$ of (3.6) by (1.11). We get

$$
\lambda=L_{P} \Omega+\frac{1}{2} \omega \wedge \theta,
$$

whence

$$
e^{-\sigma} K_{X(J X)}^{\prime}=K_{X(J X)}-\left|\left(\nabla_{X} J\right)(X)\right|^{2}|X|^{-4}-\left(L_{B} \Omega\right)(X, J X) / 2|X|^{2},
$$

which clearly proves (ii) and, in view of Theorems 8.1, 8.2 of [2], it also proves (iii). 
Corollary 3.3. Let $M$ be an l.c.K. manifold with absolutely parallel Lee form. Then, if the local metrics $g^{\prime}$ have nonnegative h.s.c., the metric $g$ also has nonnegative h.s.c. Particularly, this is true for the Hopf manifolds.

Proof. It follows from [6] that for such manifolds $L_{B} \Omega=0$ so that the statement follows from part (ii) of Theorem 3.2.

ReMarK. By (3.10), we have for the Hopf manifolds,

$$
K_{X(J X)}=\left|\left(\nabla_{X} J\right)(X)\right|^{2}|X|^{-4},
$$

and since these are not simply connected, it follows that the inequality of [2, Theorem 8.3] cannot be strengthened by $\geqslant 0$.

More information can be obtained for those 1.c.K. manifolds for which the local Kähler metrics $g^{\prime}$ have constant h.s.c. Namely, we have

TheOReM 3.4. Let $M$ be a connected l.c.K. manifold and assume that all its local Kähler metrics $g^{\prime}$ have the same constant h.s.c. $k$. Then, either $k=0$ or $M$ is globally c.K. In the first case, the supplementary hypothesis that the metric $g$ of $M$ has pointwise constant h.s.c. $\neq-|\omega|^{2} / 4$ in at least one point assures again that $M$ is globally $c . K$.

Proof. Under the hypothesis, it is known that the curvature tensor of $g^{\prime}$ is given by

$$
\begin{aligned}
R_{X Y Z W}^{\prime}=-(k / 4)\left\{g^{\prime}(X, Z) g^{\prime}(Y, W)-g^{\prime}(X, W) g^{\prime}(Y, Z)\right. \\
+g^{\prime}(X, J Z) g^{\prime}(Y, J W) \\
\left.-g^{\prime}(X, J W) g^{\prime}(Y, J Z)+2 g^{\prime}(X, J Y) g^{\prime}(Z, J W)\right\} .
\end{aligned}
$$

Together with (2.3), this gives the following expression for the curvature tensor of g.

$$
\begin{gathered}
R_{X Y Z W}=\frac{1}{2}\{L(X, Z) g(Y, W)-L(Y, Z) g(X, W) \\
+L(Y, W) g(X, Z)-L(X, W) g(Y, Z)\} \\
+\left(|\omega|^{2} / 4\right)\{g(Y, Z) g(X, W)-g(X, Z) g(Y, W)\} \\
-(k / 4) e^{-\sigma}\{g(X, Z) g(Y, W) \\
\quad-g(X, W) g(Y, Z)+\Omega(X, Z) \Omega(Y, W) \\
-\Omega(X, W) \Omega(Y, Z)+2 \Omega(X, Y) \Omega(Z, W)\} .
\end{gathered}
$$

Going over here to local coordinates by taking $X=\partial / \partial x^{i}, Y=\partial / \partial x^{j}, Z=$ $\partial / \partial x^{k}, W=\partial / \partial x^{l}$ and contracting by $g^{i k} g^{j l}$ we get

$$
n(n+1) k e^{-\sigma}=R+(2 n-1) \Lambda-n(2 n-1)\left(|\omega|^{2} / 2\right),
$$

where $R$ is the scalar curvature of $M$ and $\Lambda=g^{i j} L_{i j}$.

Formula (3.13) shows that if $k \neq 0, \sigma$ is globally defined, which proves the first statement of the theorem.

Next, suppose that $k=0$ and compute, from either (3.12) or (3.3) the holomorphic sectional curvature of $g$. We get

$$
K_{X(J X)}=|\omega|^{2} / 4+\lambda(X, J X) / 2|X|^{2} .
$$


This shows that $K_{X(J X)}$ is pointwise constant (i.e. independent of $X$ ) iff, for every $X$, one has

$$
\lambda(X, J X)=\varphi g(X, X)
$$

for some real function $\varphi$ on $M$.

Now, since $L(X, Y)$ is symmetric, we easily get

$$
\begin{aligned}
\lambda(X, Y)= & L(X, J Y)-L(J X, Y) \\
= & \frac{1}{2}\{L(X+J Y, X+J Y)-L(X, X)-L(J Y, J Y)\} \\
& +\frac{1}{2}\{L(J X-Y, J X-Y)-L(J X, J X)-L(Y, Y)\} \\
= & \frac{1}{2} \lambda(X, J X)+\frac{1}{2} \lambda(Y, J Y)-\frac{1}{2} \lambda(X+J Y, J(X+J Y)),
\end{aligned}
$$

whence we can see that $(3.15)$ holds iff

$$
\lambda(X, Y)=-\varphi \Omega(X, Y)
$$

for all $X, Y$.

I.e., in view of (3.7),

$$
d \theta-\frac{1}{2} \omega \wedge \theta=\psi \Omega \quad\left(\psi=-|\omega|^{2}-\varphi\right) .
$$

Taking in (3.17) the exterior differential, we get, because of (3.17) and rank $\Omega=$ $2 n$, that

$$
d \psi+\frac{1}{2} \psi \omega=0
$$

Locally, we always have $\omega=(2 d \tau) / \tau$ for some function $\tau \neq 0$, which implies from (3.18) that we have locally $\psi=c / \tau$ with a constant $c$. Together with the connectedness of $M$, this shows that either $\psi \equiv 0$ or $\psi \neq 0$ at every point.

In the last case we get $\omega=-(2 d \psi) / \psi$ which is a global relation and proves that $M$ is globally conformal Kähler. In the first case, we get overall $K_{X(J X)}=-|\omega|^{2} / 4$, a case which is excluded.

This ends the proof of Theorem 3.4.

REMARK. If $M$ is assumed compact, the hypothesis $K_{X(J X)} \neq-|\omega|^{2} / 4$ is no longer necessary. In fact, in the exceptional case $\psi=0$, we have by (3.17), $d \theta=\frac{1}{2} \omega \wedge \theta$, i.e., in view of (1.2), $d \delta \Omega=\frac{1}{2} e(\omega) \delta \Omega$. Here, we take the global scalar product by $\Omega$ and use also $\theta=i(\omega) \Omega$ [7]. This yields

$$
(\delta \Omega, \delta \Omega)=-(1 / 2(n-1))(\delta \Omega, \delta \Omega),
$$

whence $\delta \Omega=0$, i.e., $\theta=0$. Q.E.D.

Note. We learned later on that some properties of the manifolds in $\mathrm{CF}_{3}$ have been established by T. Kashiwada, Some properties of locally conformal Kähler manifolds (preprint).

\section{REFERENCES}

1. S. I. Goldberg, Curvature and homology, Academic Press, New York, 1962.

2. A. Gray, Curvature identities for Hermitian and almost Hermitian manifolds, Tôhoku Math. J. 28 (1976), 601-612. 
3. S. Kobayashi and K. Nomizu, Foundations of differential geometry. II, Interscience, New York, 1969.

4. P. Libermann, Sur les structures presque complexes et autres structures infinitesimales régulières, Bull. Soc. Math. France 83 (1955), 195-224.

5. I. Vaisman, On locally conformal almost Kähler manifolds, Israel J. Math. 24 (1976), 338-351.

6. ___ Locally conformal Kähler manifolds with parallel Lee form, Rend. Mat. (to appear).

7. L__ Remarkable operators and commutation formulas on locally conformal Kähler manifolds, Compositio Math. (to appear).

Department of Mathematics, University of Haifa, Mt. Carmel, Hafa (31999), Israrl 